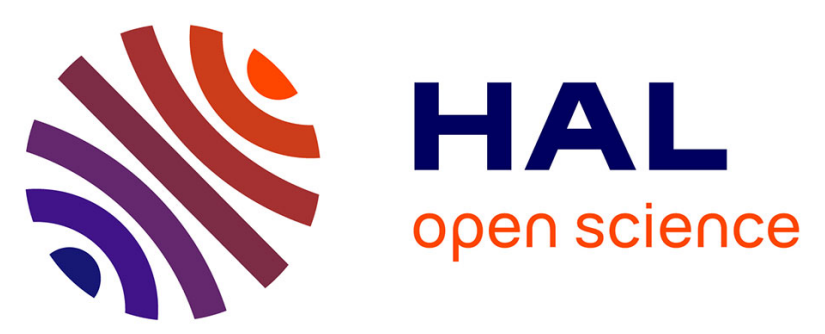

\title{
OFDM-based Spatial Data Focusing for High Resolution 2-Dimensional Wireless Geocasting
}

Guylian Molineaux, Michael Odhiambo, Francois Horlin, Philippe de Doncker, Julien Sarrazin

\section{- To cite this version:}

Guylian Molineaux, Michael Odhiambo, Francois Horlin, Philippe de Doncker, Julien Sarrazin. OFDM-based Spatial Data Focusing for High Resolution 2-Dimensional Wireless Geocasting. Conference PIMRC 2020, Aug 2020, Londres, United Kingdom. pp.1-6, 10.1109/PIMRC48278.2020.9217222 . hal-03151943

\section{HAL Id: hal-03151943 \\ https://hal.sorbonne-universite.fr/hal-03151943}

Submitted on 25 Feb 2021

HAL is a multi-disciplinary open access archive for the deposit and dissemination of scientific research documents, whether they are published or not. The documents may come from teaching and research institutions in France or abroad, or from public or private research centers.
L'archive ouverte pluridisciplinaire HAL, est destinée au dépôt et à la diffusion de documents scientifiques de niveau recherche, publiés ou non, émanant des établissements d'enseignement et de recherche français ou étrangers, des laboratoires publics ou privés. 


\title{
OFDM-based Spatial Data Focusing for High Resolution 2-Dimensional Wireless Geocasting
}

\author{
Guylian Molineaux $^{* \dagger}$, Michael Odhiambo ${ }^{\dagger *}$, François Horlin*, Philippe De Doncker* and Julien Sarrazin ${ }^{\dagger}$ \\ * OPERA - Wireless Communications Group \\ Université Libre de Bruxelles, 1050 Brussels, Belgium \\ ${ }^{\dagger}$ CNRS, Laboratoire de Genie Electrique et Electronique de Paris \\ Sorbonne Université, 75005 Paris, France \\ Univ. Paris-Saclay, CentraleSupélec, 91192 Gif-sur-Yvette, France
}

\begin{abstract}
An OFDM-based Spatial Data Focusing (OFDMSDF) approach is proposed as an improvement over standard Time-based Spatial Data Focusing (T-SDF) as a means of wirelessly broadcasting information towards confined spatial locations, i.e. wireless geocasting. It is shown that this approach allows for 2-dimensional focusing, hence leading to far greater flexibility in terms of geocasting scenarios compared to T-SDF and beamforming, both limited to angular focusing only. This increased flexibility does not come at any trade-off costs in terms of spatial selectivity, and hence, just as T-SDF, OFDMSDF is shown to establish a considerable increase in attainable geocasting accuracy when compared to traditional power focusing methods. This paper describes the free space OFDM-SDF system model for uniform linear antenna arrays, including beamsteering and sidelobe mitigation techniques. Based on simulations, the performance of OFDM-SDF is compared to both T-SDF, as well as classical beamforming.
\end{abstract}

Index Terms-Geocasting, Spatial Data Focusing, Orthogonal Frequency-Division Multiplexing, Wireless Communications

\section{INTRODUCTION}

Geocasting, proposed in [1] as a location-based multicasting scheme, ensures that transmitted information is available to users in a designated target area only and inaccessible to users located elsewhere. It does so without requiring knowledge at the base station of a user's presence in this area, hence avoiding potential privacy concerns. This transmission scheme can provide great opportunities in the scope of the Internet of Things and Smart City environments, where large groups of mobile devices could benefit from receiving location relevant or contextualized information, e.g. for tourism, navigation, marketing, or management purposes, without the necessity to share their location. If one wants to eliminate additionally the requirement of self-localizing nodes and multihop forwarding techniques, as required in [1], information can be wirelessly transmitted from fixed base stations towards all nodes within their coverage area. Geocasting in this scenario is possible, on the condition that base stations exhibit spatial focusing capabilities towards certain target areas.

This work was supported by the ANR GEOHYPE project, grant ANR-16CE25-0003 of the French Agence Nationale de la Recherche, and carried out in the framework of COST Action CA15104 IRACON. G. Molineaux is a FRIA grantee of the Fonds de la Recherche Scientifique - FNRS.

978-1-7281-4490-0/20/\$31.00 @ 2020 IEEE
Beamforming is a traditional approach employed to realize the above introduced scenario. It uses phased arrays, exploiting constructive and destructive interference such that power is spatially focused towards a certain area [2]. As a result, correct signal recovery is only possible in the restricted areas where the signal-to-noise ratio (SNR) is sufficient. Beamforming is however inherently limited in applicability for geocasting scenarios, in the sense that large arrays are required to create narrow beams, i.e. to accurately target a certain area. Additionally, beamforming suffers from sidelobes, where the SNR is lower than in the main lobe, however potentially sufficiently high for sensitive receivers to recover the data in areas other than the intended ones.

In the context of physical layer security, the latter shortcoming can be improved upon by the use of Directional Modulation (DM) techniques, essentially a symbol-level precoding extension of beamforming. Traditionally, this is either performed directly at RF level through the manipulation of the near-field radiation pattern of the array elements [3], [4], or, alternatively and more closely related to beamforming, unique symbol-specific weighing vectors can be used to ensure scrambling of data in all but one direction [5], [6]. While these approaches succeed in securing beamforming from eavesdropping in sidelobe direction, they typically don't yield considerable improvements in terms of beamwidth and geocasting accuracy. Certain alternative DM implementations, like [7], have shown on the other hand to provide significant beamwidth improvements over beamforming. They require however accurate time synchronization between widely spaced antennas, such that large bandwidths are required and the necessary physical size of the array remains large.

By removing the constraint on the array radiation pattern, Spatial Data Focusing (SDF) allows to improve upon the performance of both aforementioned techniques. Instead, SDF omnidirectionally transmits uncorrelated orthogonal signals over the different transmitting antennas in a multiple-input single-output (MISO) channel, each carrying part of a global datastream. It then exploits, at the receiver, the different propagation conditions of each data substream to induce an intentional and location-dependent distortion of the symbol 
constellation on one or multiple substreams. Correct recovery of the full datastream, i.e. low bit-error-rate (BER), is so only possible when all substreams are correctly recovered, which, by design of the SDF system, occurs in spatially restricted areas only. Due to the lack of power focusing, SDF's spatial selectivity is not assessed using the traditional halfpower beamwidth, instead the beamwidth is defined as the geographical area where the BER is below a certain threshold.

In [8] and [9], SDF was first introduced by exploiting the temporal dimension to ensure signal orthogonality. This Time-based Spatial Data Focusing (T-SDF) implementation modulates the different data substreams onto time-shifted waveforms and transmits them sequentially in orthogonal time slots. While this approach clearly shows the potential of SDF in improving geocasting accuracy over beamforming, it is limited, as is beamforming itself, by its inability to focus according to range, hence limiting geocasting applicability.

OFDM-based Spatial Data Focusing (OFDM-SDF), as proposed in this paper, exploits the OFDM subcarrier orthogonality to introduce signal orthogonality in the SDF system model. The multi-frequency transmission of signals gives rise to an additional degree of freedom in the SDF system that allows to perform 2-dimensional focusing, i.e. both in the angular and range domains, while simultaneously preserving the improvements in terms of geocasting accuracy as established by T-SDF.

Exploitation of multi-frequency transmission benefits has been explored in the context of range-angle-dependent beamforming as well, specifically through Frequency Diverse Arrays (FDAs) [10]. The obtained beampatterns are however coupled in the range and angular domains, resulting in 2dimensional, yet continuous beampattern curves. OFDM-SDF on the other hand allows to create isolated regions of low BER, hence successfully restricting the geocast target area to a 2-dimensionally confined region.

While Random Frequency Diverse Arrays (RFDAs) allow to decouple range and angular domains in beamforming [11], they require however stochastic description of their beampatterns and are mostly suited for active sensing in radar applications rather than geocasting. In DM context, RFDAs have shown to achieve desirable 2-dimensional focusing capability [12]. Spatial selectivity is however obtained by manipulation of the signal-to-interference-plus-noise ratio (SINR) through the addition of artificial noise (AN) in an eavesdropper's steering space, an operation that is not required in SDF approaches.

Section II of this paper introduces the free space system model used for OFDM-SDF. Section III expands this model to allow for beamsteering towards an arbitrary user-specified location, after which Section IV derives a set of design rules that ensure the uniqueness of the area where data is retrievable. Finally, after studying the performance based on simulations in Section V, conclusions are drawn in Section VI.

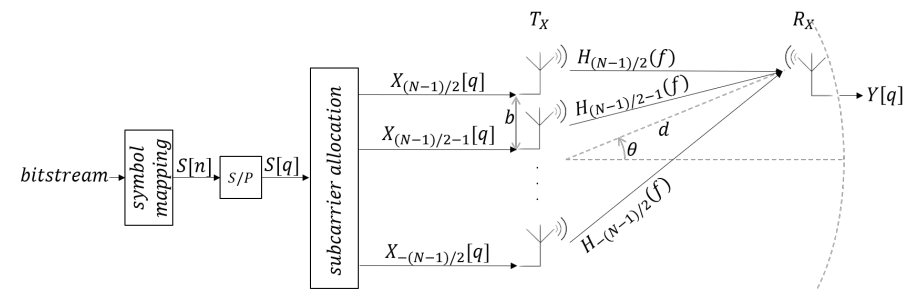

Fig. 1. Frequency domain OFDM-SDF free space system model

\section{Free Space System Model}

Fig. 1 shows the frequency domain system model that is considered for OFDM-SDF in a free space scenario. A uniform linear array of $N$ antennas is used, with element spacing $b$. Taking the middle of the array as the origin, the antenna coordinates along the array axis are given by $i b$, where $i \in\{-(N-1) / 2,-(N-1) / 2+1, \ldots,(N-1) / 2\}$ is the index indicating the antenna in the array and its corresponding channel. In the context of OFDM, $Q$ subcarriers are considered to be equally and continuously spaced in a frequency band $B$, yielding a subcarrier bandwidth $B_{c}=B / Q$. A receiver is assumed to move in a 2-dimensional plane, with a position expressed in polar coordinates $(d, \theta)$. Where $d$ represents the radial distance between the array center and the receiver, and $\theta$ the azimuth angle of the receiver with respect to the array broadside direction.

\section{A. Transmitter-side signal processing}

At the transmitter, an arbitrary bitstream is mapped onto a linear symbol stream $S[n]$, using any desired mapping scheme. As in traditional OFDM, this symbol stream is converted from serial to parallel, yielding OFDM blocks $S[q, u]$, where the indices $q$ and $u$ represent respectively the OFDM subcarrier index and the OFDM block index. Without loss of generality, only a single OFDM block can be considered and hence the index $u$ is omitted in the remainder of this paper. The assumption of continuous and equal spacing of subcarriers leads to a set of possible values for $q$, given by $\mathcal{Q}=\{-Q / 2+1 / 2,-Q / 2+3 / 2, \ldots, Q / 2-1 / 2\}$, allowing to express the subcarrier frequency $f_{q}$ with respect to the carrier frequency $f_{c}$ as $f_{q}=f_{c}+q B_{c}$. Typical for SDF, information is transmitted in a distributed way over the $N$ antennas in the array, using orthogonal signals to avoid interference between the different data substreams. Specifically for OFDM-SDF, orthogonality is introduced by exploiting the orthogonal nature of OFDM subcarriers. To this end, a subset of subcarriers $\mathcal{Q}_{i} \subset \mathcal{Q}$ is assigned to each antenna $i$, in such a way that each subcarrier in $\mathcal{Q}$ is assigned exclusively to one of the subsets $\mathcal{Q}_{i}$. A new antenna-specific OFDM block $X_{i}$ is then defined for each antenna $i$ based on the subcarriers allocated to this antenna

$$
X_{i}[q]= \begin{cases}S[q] & q \in \mathcal{Q}_{i} \\ 0 & q \in \mathcal{Q} \backslash \mathcal{Q}_{i}\end{cases}
$$


In other words, the antenna specific OFDM block $X_{i}$ is identical to the initial OFDM block $S$, on those subcarriers that are assigned to the $i$-th antenna and contains no information on all other subcarriers. Equivalently, but more compactly, in matrix notation, one finds that

$$
X_{i}=W_{i}^{\text {alloc }} S,
$$

where $W_{i}^{\text {alloc }}$ is a $Q \times Q$ matrix, containing ones only on those diagonal elements with row and column indices corresponding to the carrier indices in $\mathcal{Q}_{i}$, and zeroes elsewhere.

While subcarrier allocation is arbitrary in the most general scenario and the validity of the OFDM-SDF concept is preserved regardless of the adopted allocation convention, for simplicity of the derivations that follow however, this paper assumes that subsequent subcarriers are assigned in an alternating way to the different antennas. Specifically, the first subcarrier in $\mathcal{Q}$ is allocated to either one of the outer antennas, after which the following subcarriers are assigned to the other antennas in the order that starts at the antenna closest to the first one, until reaching the antenna at the opposite end of the array. This pattern is repeated until all subcarriers have been assigned to an antenna.

\section{B. Free space MISO propagation channel influences}

Each OFDM block $X_{i}$ is transmitted from a different antenna in the MISO setup and hence undergoes distinctive propagation conditions. Specifically, for a free space scenario, each channel $i$ is characterized by a unique propagation delay $\tau_{i}$, while it can be assumed that the channel attenuation coefficients and channel phases are identical for all channels (denoted respectively as $a$ and $\phi$ ). As such, the channel transfer function corresponding to the $i$-th channel is given by

$$
H_{i}(f)=a e^{j \phi} e^{-j 2 \pi\left(f_{c}+f\right) \tau_{i}}
$$

where $f$ represents the baseband frequency.

Under the assumption that the channel coherence bandwidth is larger than the subcarrier bandwidth, by default satisfied in a free space scenario, the channel transfer function $H_{i}(f)$ is constant in the frequency band of each subcarrier $q$, where its value is given by $H_{i}[q]=H_{i}\left(q B_{c}\right)$. As a result, the expression for the received symbol from the $q$-th subcarrier in the $i$-th channel is given by

$$
\begin{aligned}
Y_{i}[q] & =H_{i}[q] X_{i}[q]=a X_{i}[q] e^{j \phi} e^{-j 2 \pi\left(f_{c}+q B_{c}\right) \tau_{i}} \\
& = \begin{cases}a S[q] e^{j \phi} e^{-j 2 \pi\left(f_{c}+q B_{c}\right) \tau_{i}} & q \in \mathcal{Q}_{i} \\
0 & q \in \mathcal{Q} \backslash \mathcal{Q}_{i} .\end{cases}
\end{aligned}
$$

The total received OFDM block $Y$ is, evidently, the sum of all OFDM blocks $Y_{i}$ and will hence contain a single received symbol on each of the subcarriers $q \in \mathcal{Q}$. Each symbol being influenced by the channel $i$, to which the subcarrier was assigned.

\section{Receiver-side signal processing and channel equalization}

OFDM-SDF's multi-frequency transmission, allows to exploit an additional degree of freedom in the SDF equalization process, in addition to the propagation delay differences exploited in T-SDF. To this end, instead of defining a single reference channel as in [9], a set of reference subcarriers $\mathcal{Q}_{\text {ref }}$ is defined to be used for channel estimation and equalization. While the choice of this set is free in general, the creation of a 2-dimensionally isolated target area requires that a pair of two reference channels $H_{r e f 1}$ and $H_{r e f 2}$, respectively with assigned subcarrier sets $\mathcal{Q}_{\text {ref } 1}$ and $\mathcal{Q}_{\text {ref } 2}$, is chosen such that they comply to the following specifications:

- Primary reference channel, $H_{r e f 1}$ : Channel corresponding to the outermost antenna in the array, with negative index, i.e. $i_{\text {ref } 1}=-(N-1) / 2 .^{1}$

- Complementary reference channel, $H_{\text {ref } 2}$ : Channel corresponding to the antenna opposite, w.r.t. the array center, to the primary reference antenna, i.e. $i_{r e f 2}=-i_{r e f 1}$.

The set of reference subcarriers is then constructed as

$$
\mathcal{Q}_{\text {ref }}=\left\{\mathcal{Q}_{\text {ref } 1} \mid q<0\right\} \cup\left\{\mathcal{Q}_{\text {ref } 2} \mid 0<q\right\},
$$

i.e. in the lower half of the total bandwidth, reference subcarriers are taken from the primary reference channel's subcarrier set, while they are taken from the complementary reference channel's subcarrier set in the upper half of the total bandwidth.

Channel estimation is performed only for the subchannels corresponding to the subcarriers present in the reference set $\mathcal{Q}_{\text {ref }}$. Specifically, the channel estimation $\hat{H}^{S D F}$ used for OFDM-SDF equalization purposes is defined as

$$
\hat{H}^{S D F}[q]=\left\{\begin{array}{l}
\hat{H}_{\text {ref }}[q]=a e^{j \phi} e^{-j 2 \pi\left(f_{c}+q B_{c}\right) \tau_{\text {ref }}} \\
\hat{H}_{\text {ref }}\left[q_{\text {ref }}\right] \\
q \in \mathcal{Q}_{\text {ref }}
\end{array}\right.
$$

where the index $q_{\text {ref }}$ refers to the reference subcarrier that is closest to, but smaller than, the subcarrier $q$ that is to be equalized. In other words, the channel estimation $\hat{H}^{S D F}$ is equal to the estimation $\hat{H}_{\text {ref }}$ of one of the reference channels on the subcarriers that belong to the reference set, and is, for non-reference subcarriers, equal to a copy of the reference channel estimation on the previous reference subcarrier. ${ }^{2}$

The following notations are used to express the differences between the subchannel corresponding to the $q$-th subcarrier on the $i$-th channel and the subchannel corresponding to a reference subcarrier $q_{\text {ref }} \in \mathcal{Q}_{\text {ref }}$ on the corresponding reference channel. Analogous to T-SDF, $\Delta \tau_{i}$ expresses the

\footnotetext{
${ }^{1}$ In the most general case, the choice of the primary reference channel is arbitrary, however the adopted conventions both simplify the analysis that follows and maximize angular selectivity.

${ }^{2}$ Note that, for the remainder of this paper, the term reference channel can refer to either the primary or the complementary reference channel. Whether the former of the latter should be employed is implied by the subcarrier range that is considered, in accordance to (5).
} 
relative difference in propagation delay, between the $i$-th channel and the reference channel, i.e. $\tau_{i}=\tau_{\text {ref }}+\Delta \tau_{i}$. Additionally, for OFDM-SDF with equal and continuous subcarrier spacing, the frequency difference between a subcarrier $q$ and a reference subcarrier $q_{\text {ref }}$ is given by $\Delta q B_{c}$, such that $q B_{c}=\left(q_{r e f}+\Delta q\right) B_{c}$, with $\Delta q \in \mathbb{N}$.

Using these conventions, traditional Zero Forcing OFDM equalization of the symbol $Y_{i}[q]$, received from the $q$-th subcarrier in the $i$-th channel, yields the following expression for the equalized symbol from the same channel and subcarrier

$$
\begin{aligned}
\hat{Y}_{i}[q] & =Y_{i}[q] / \hat{H}^{S D F}[q] \\
& = \begin{cases}S[q] e^{-j 2 \pi\left(f_{c}+q B_{c}\right) \Delta \tau_{i}} e^{-j 2 \pi \Delta q B_{c} \tau_{r e f}} & q \in \mathcal{Q}_{i} \\
0 & q \in \mathcal{Q} \backslash \mathcal{Q}_{i} .\end{cases}
\end{aligned}
$$

\section{Spatial restrictions on correct data retrieval}

Correct data retrieval is possible on the condition that the residual phase shift, present on the equalized symbols in (7b), is equal to an integer multiple of $2 \pi$, i.e.

$$
-2 \pi\left(f_{c}+q B_{c}\right) \Delta \tau_{i}-2 \pi \Delta q B_{c} \tau_{r e f}=k 2 \pi, \quad k \in \mathbb{Z} .
$$

Based on the paraxial approximation $(b \ll d)$, the parameters $\Delta \tau_{i}$ and $\tau_{r e f}$ can be substituted by equivalent expressions

$$
\begin{gathered}
\Delta \tau_{i}=-\Delta i \frac{b}{c} \sin \theta, \\
\tau_{\text {ref }}=\frac{d}{c}-i_{\text {ref }} \frac{b}{c} \sin \theta .
\end{gathered}
$$

Where $c$ is the speed of light and $\Delta i=i-i_{\text {ref }}$ represents the difference in index between the equalized and reference channel. This allows to derive an expression describing the radial distance $d$ at which data is correctly received, as a function of the receiver azimuth position $\theta$

$$
d=\frac{c}{\Delta q B_{c}} k+\left(\frac{\Delta i}{\Delta q} \frac{f_{c}+q B_{c}}{B_{c}}+i_{r e f}\right) b \sin \theta, \quad \Delta q \neq 0 .
$$

This expression is simplified by noting that: (i) the combined choice of reference channels at the outer antennas and an alternating subcarrier allocation scheme (as defined in Section II-A) ${ }^{3}$ yields $\Delta i=-\operatorname{sgn}\left(i_{r e f}\right) \Delta q$, (ii) OFDM uses narrowband subcarriers (i.e. $q, i_{r e f} \ll f_{c} / B_{c}$ ), and (iii) all curves (10) (i.e. for all channels; equivalently: for all values of $\Delta q$ ) should coincide to ensure correct recovery of the full datastream, which occurs only in the loci described by (10) with $\Delta q=1$

$$
d \approx \frac{c}{B_{c}} k-\operatorname{sgn}\left(i_{r e f}\right) \frac{f_{c}}{B_{c}} b \sin \theta .
$$

By using a pair of reference channels $H_{\text {ref } 1}$ and $H_{\text {ref } 2}$, two of the above patterns, $d_{1}$ and $d_{2}$, are created and the resulting BER is low only at their intersection(s), i.e.

$$
d_{1}=d_{2} \Longleftrightarrow \frac{c}{B_{c}} k_{1}+\frac{f_{c}}{B_{c}} b \sin \theta=\frac{c}{B_{c}} k_{2}-\frac{f_{c}}{B_{c}} b \sin \theta
$$

${ }^{3}$ Note that the alternating subcarrier allocation should be performed separately in the two different subcarrier ranges that correspond to each of the reference channels.
Where the subscripts 1 and 2 refer respectively to the primary and complementary reference channels.

After development of this expression, one finds that data can be correctly retrieved at azimuth angles corresponding to

$$
\theta=\arcsin \left(\frac{c}{2 f_{c} b}\left(k_{2}-k_{1}\right)\right)=\arcsin \left(\frac{1}{2 \alpha} k^{\prime}\right),
$$

where the last equality is obtained by writing the antenna spacing as a fraction $\alpha$ of the carrier wavelength $\lambda_{c}$, i.e. $b=\alpha \lambda_{c}$, and replacing the difference of two integers $k_{2}-k_{1}$, by a substitute integer $k^{\prime}$. The coordinates of the locations in which errorless communication is possible are given by substituting the solutions of (13) into (11).

\section{BEAMSTEERING}

While the approach described in Section II provides the description of a fully functional OFDM-SDF system in free space, it is restricted by the fact that the locations of correct data retrieval cannot be actively manipulated. By adding at the transmitter side, on each subcarrier $q$ and each antenna $i$, a unique steering phase $\varphi_{i, q}^{\text {steer }}$, it will be shown that the location of low BER can be altered to any arbitrary position $\left(d^{\text {steer }}, \theta^{\text {steer }}\right)$. To this end, definition (1) of the antennaspecific OFDM blocks is expanded to include the influence of the steering phases, i.e.

$$
X_{i}[q]= \begin{cases}S[q] e^{j \varphi_{i, q}^{\text {steer }}} & q \in \mathcal{Q}_{i} \\ 0 & q \in \mathcal{Q} \backslash \mathcal{Q}_{i} .\end{cases}
$$

The steering operation can easily be included in the matrix model introduced in Section II by defining a $Q \times Q$ matrix $W_{i}^{\text {steer }}$, which contains on the diagonal elements the steering phases of the $i$-th antenna with subcarrier index corresponding to the row and column index of the respective diagonal element, and zeros elsewhere. One finds

$$
X_{i}=W_{i}^{\text {steer }} W_{i}^{\text {alloc }} S .
$$

Using an analogous reasoning as in Section II, one finds as an expression for the equalized symbols, received from the $q$-th subcarrier in the $i$-th channel, in the presence of beamsteering phases,

$$
\hat{Y}_{i}[q]=\left\{\begin{array}{rr}
S[q] e^{j \Delta \varphi_{i, q}^{\text {steer }}} e^{-j 2 \pi\left(f_{c}+q B_{c}\right) \Delta \tau_{i}} e^{-j 2 \pi \Delta q B_{c} \tau_{r e f}} \\
q \in \mathcal{Q}_{i} \\
0 & q \in \mathcal{Q} \backslash \mathcal{Q}_{i} .
\end{array}\right.
$$

This result is of course identical to the result obtained in Section II, with the addition of a phase shift $\Delta \varphi_{i, q}^{\text {steer }}=\varphi_{i, q}^{\text {steer }}-\varphi_{\text {ref }, q}^{\text {steer }}$, depending on the difference in steering phase between the equalized channel and the reference channel.

Once again, correct retrieval of the transmitted symbols is only possible on the condition that the residual phase shift, present in (16), is equal to an integer multiple of $2 \pi$, i.e.

$\Delta \varphi_{i, q}^{\text {steer }}-2 \pi\left(f_{c}+q B_{c}\right) \Delta \tau_{i}-2 \pi \Delta q B_{c} \tau_{r e f}=k 2 \pi, \quad k \in \mathbb{Z}$. 
Further development of this statement yields an expression for the steering phase shift to add to the symbols transmitted on the $q$-th subcarrier from the $i$-th channel

$$
\varphi_{i, q}^{\text {steer }}=2 \pi\left[k+\left(f_{c}+q B_{c}\right) \Delta \tau_{i}+\Delta q B_{c} \tau_{r e f}\right]+\varphi_{\text {ref }, q}^{\text {steer }}
$$

The integer $k$ adds or subtracts only multiples of $2 \pi$ to or from the steering phase and hence it can be arbitrarily chosen as zero. Additionally, by (16), clearly only the relative difference in steering phase with respect to the reference subcarrier is relevant and, as a result, $\varphi_{\text {ref, } q}^{\text {steer }}$ can be arbitrarily chosen to be zero as well. Finally, after substituting again $\Delta \tau_{i}$ and $\tau_{\text {ref }}$ by their equivalent expressions (9a) and (9b), the final expression for the steering phases can be found as

$$
\begin{aligned}
\varphi_{i, q}^{\text {steer }}=2 \pi[ & -\left(f_{c}+q B_{c}\right) \frac{b}{c} \Delta i \sin \theta^{\text {steer }} \\
& \left.+\frac{\Delta q B_{c}}{c}\left(d^{\text {steer }}-i_{r e f} b \sin \theta^{\text {steer }}\right)\right] .
\end{aligned}
$$

\section{Sidelobe Mitigation}

In the presence of steering phases, (11) and (13) are transformed to, respectively,

$$
\begin{gathered}
d \approx \frac{c}{B_{c}}\left(k+\frac{\varphi_{i^{*}, q^{*}}^{\text {steer }}}{2 \pi}\right)-\operatorname{sgn}\left(i_{r e f}\right) \frac{f_{c}}{B_{c}} b \sin \theta \\
\theta=\arcsin \left(\frac{1}{2 \alpha}\left(k^{\prime}+\frac{\varphi_{i_{2}^{*}, q_{2}^{*}}^{\text {steer }}-\varphi_{i_{1}^{*}, q_{1}^{*}}^{\text {ster }}}{2 \pi}\right)\right) .
\end{gathered}
$$

Where, analogous to (11), the expression for $d$ is obtained by observing the subcarriers, and their corresponding channels, with $\Delta q=1$ (respectively $\Delta i= \pm 1$ ), which is indicated by a superscript $*$ in the steering phase indices.

The above result leads to a solution and hence an area where data is correctly received, when the argument of the inverse sine function in (20b) lies within the interval $[-1,1]$. Using the same assumptions as in the derivation of (11), (19) yields that $\left(\varphi_{i_{2}^{*}, q_{2}^{*}}^{\text {steer }}-\varphi_{i_{1}^{*}, q_{1}^{*}}^{\text {steer }}\right) / 2 \pi=2 \alpha \sin \theta^{\text {steer }}$. As a result, whatever the value of the steering phases $\varphi_{i_{1}^{*}, q_{1}^{*}}^{\text {steer }}$ and $\varphi_{i_{2}^{*}, q_{2}^{*}}^{\text {ster }}$, when $\alpha$ is bounded by $1 / 2$, compliance to this condition is ensured for $k^{\prime}=0$. This solution corresponds to the area of low BER that will be created around the intended steering point. If, however, solutions for (20b) exist for $k^{\prime} \neq 0$, undesired spurious locations of low BER will exist at azimuth angles other than the intended one. Hence, the formal condition that ensures mitigation of sidelobes in the angular domain is given by

$$
\left|\frac{1}{2 \alpha}\left(k^{\prime}+2 \alpha \sin \theta^{\text {steer }}\right)\right|>1, \quad \forall k^{\prime} \in \mathbb{Z}_{0} .
$$

Keeping only the positive bounds on $\alpha$ and noting that imposing $\left|k^{\prime}\right|=1$ yields the most strict upper bound, one finds that angular sidelobes are avoided when

$$
\alpha<\frac{1}{2} \frac{1}{1+\left|\sin \theta^{\text {steer }}\right|} .
$$

One can see that, as anticipated, $\alpha<1 / 2$ is enforced regardless of the steering angle $\theta^{\text {steer }}$. It should be noted that the above approximations, used to derive (20a), (22), and $\left(\varphi_{i_{2}^{*}, q_{2}^{*}}^{\text {steer }}-\varphi_{i_{1}^{*}, q_{1}^{*}}^{\text {steer }}\right)$, are justified in the sense that, in practice, at all times, an area of spread out low BER will be present around any spurious spot. As a result, the practical bound on $\alpha$ should always be chosen slightly lower than the theoretical one, such that approximate knowledge of the latter is sufficient.

Just like (11), for a fixed angle $\theta$, (20a) is periodic in the range domain, with periodicity $c / B_{c}$, hence leading to spurious locations along the radial axis, where data is, but should not be, retrievable. Spurious spots of low BER along the radial axis are unavoidable, due to the infinite character of this dimension. However, in practice, if one can ensure that no spurious spot is present between the base station and the desired steering point, and additionally that the first spurious spot occurring after the steering point falls beyond a range $d^{a t t}$ where signal recovery is prohibited by sufficient attenuation, spurious spots along the radial axis can be considered to be absent. This reasoning leads to the following condition to avoid spurious locations of low BER in the distance domain

$$
B_{c}<\min \left\{\frac{c}{d^{\text {steer }}}, \frac{c}{d^{\text {att }}-d^{\text {steer }}}\right\} \text {. }
$$

Equations (19), (22), and (23) provide a set of design rules that allows one to design a full OFDM-SDF system, ensuring correct retrieval of transmitted data in an arbitrarily specified focusing location only, in the absence of any spurious locations where data might otherwise be correctly received as well.

\section{Simulations and Performance Evaluation}

Simulations of an OFDM-SDF system, including beamsteering and sidelobe mitigation processes, are performed using the following parameters. Steering coordinates are chosen as $\left(d^{\text {steer }}=250 \mathrm{~m}, \theta^{\text {steer }}=20^{\circ}\right)$. Compliance to (22) and (23) is ensured by setting respectively $\alpha=0.325$ and $B_{c}=781.25 \mathrm{kHz}$ (with $B=100 \mathrm{MHz}$ and $Q=128$ ). The number of antennas is chosen as $N=2$ and a carrier frequency of $f_{c}=3.6 \mathrm{GHz}$ is employed. The transmitted bitstream is of length $10^{5}$, it is mapped onto 16 QAM symbols. The SNR is fixed at $25 \mathrm{~dB}$ for all receiver positions.

Fig. 2 illustrates the 2-dimensional spatial BER distribution that is obtained using the above described parameters and setup. The array is aligned with the $y$-axis and centered around the origin. The red dot in the figure indicates the exact location of the desired focus point. As one can see, the area in which the BER is low, and hence where data is accessible, is centered exclusively around this position, in the absence of any sidelobes.

Evaluation of the beamwidth and accuracy along the azimuth and radial axes is done using Fig. 3 and 4. They show respectively an intersection of the BER pattern from Fig. 2 at the steering distance $d^{\text {steer }}$ and the steering angle $\theta^{\text {steer }}$. The red dotted line in both figures shows the respective intended steering coordinates. 

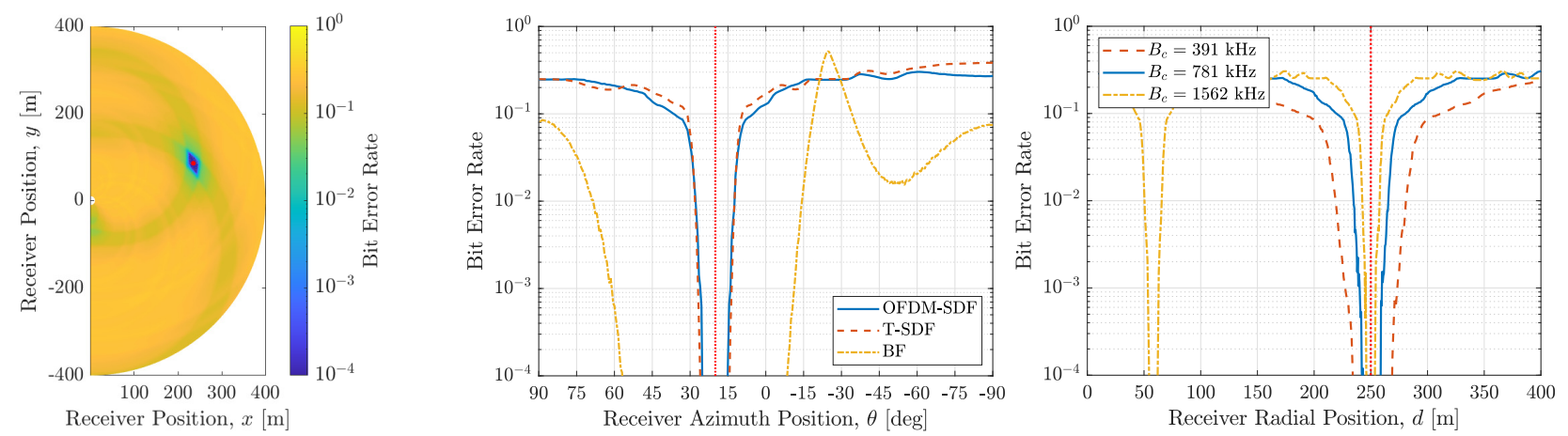

Fig. 2. 2D BER pattern for OFDM-SDF $\left(f_{c}=\right.$ Fig. 3. Intersection of OFDM-SDF BER pattern Fig. 4. Intersection of OFDM-SDF BER pattern $3.6 \mathrm{GHz}, N=2, B_{c}=781.25 \mathrm{kHz}, b=$ at $d^{\text {steer }}$ $0.325 \lambda_{c}, S N R=25 \mathrm{~dB}$ ) at $\theta^{\text {steer }}$

The former demonstrates that OFDM-SDF exhibits no tradeoff in terms of angular selectivity compared to T-SDF, as both techniques display the same beamwidth. As a consequence, angular focusing resolution can be manipulated in similar ways, as described in [9]. A BER pattern obtained when performing beamforming (BF) (i.e. feeding identical but phase shifted input signals, containing all subcarriers, to a uniform linear phased array, targeting the same angle $\theta^{\text {steer }}$ ) is shown, to illustrate the increase in angular selectivity that SDF exhibits. The number of array elements for beamforming was increased to $N=4$ since a 2 -antenna array with a spacing factor of $\alpha=0.325$ yields insufficient power focusing to create any region of low BER. Noise is added in the beamforming scenario such that the same SNR as in the SDF scenarios is obtained in the main lobe. In other directions the received power and hence the SNR and BER vary according to the array radiation pattern, yielding the BER curve in Fig. 3.

The latter illustrates that the spatial selectivity along the radial axis can be modified by adjusting the subcarrier bandwidth (steering phases $\varphi_{i, q}^{\text {steer }}$ are adjusted accordingly to maintain the same focus distance $d^{\text {steer }}$ ). Specifically, higher subcarrier bandwidths yield a higher precision, and vice versa. This is in correspondence with (16), where clearly higher subcarrier bandwdiths cause larger residual phases at equal delays $\tau_{r e f}$, i.e. at equal distances $d_{r e f}$. Increasing the range-based focusing accuracy of OFDM-SDF, does come however at the cost of introducing sidelobes along the radial axis when (23) is not satisfied, as anticipated in Section IV.

\section{Conclusion and Perspectives}

OFDM-based Spatial Data Focusing (OFDM-SDF) was introduced as an alternative for previously studied Time-based Spatial Data Focusing (T-SDF). It was shown, after a formal description of a free space OFDM-SDF system model, that no decrease in angular selectivity is present when using OFDMSDF instead of T-SDF. Hence, OFDM-SDF possesses all the main advantages that T-SDF established over beamforming and directional modulation, most importantly being the increased spatial selectivity and simple transmitter architectures. Additionally, OFDM-SDF stands out by the ability to perform 2-dimensional focusing, i.e. in both the angular as well as the radial domain, and hence provides greater flexibility in terms of possible geocasting scenarios. Furthermore, a set of design rules was developed, allowing focusing to an arbitrary user-specified position in a 2-dimensional plane, including the mitigation of all spurious locations where information might otherwise be retrievable.

\section{REFERENCES}

[1] Y.-B. Ko and N. H. Vaidya, "Geocasting in mobile ad hoc networks: location-based multicast algorithms," in Proceedings WMCSA'99. Second IEEE Workshop on Mobile Computing Systems and Applications, New Orleans, LA, USA, Feb. 1999, pp. 101-110.

[2] C. A. Balanis, Antenna Theory, analysis and design, 3rd ed. Hoboken, New Jersey, USA: John Wiley \& Sons, Inc., 2005, ch. 6, pp. 285-384.

[3] A. Babakhani, D. B. Rutledge, and A. Hajimiri, "Transmitter architectures based on near-field direct antenna modulation," IEEE Journal of Solid-State Circuits, vol. 43, no. 12, pp. 2674-2692, Dec. 2008.

[4] M. P. Daly and J. T. Bernhard, "Beamsteering in pattern reconfigurable arrays using directional modulation," IEEE Transactions on Antennas and Propagation, vol. 58, no. 7, pp. 2259-2265, Jul. 2010.

[5] Y. Ding and V. Fusco, "Orthogonal vector approach for synthesis of multi-beam directional modulation transmitters," IEEE Antennas and Wireless Propagation Letters, vol. 14, pp. 1330-1333, Feb. 2015.

[6] M. P. Daly and J. T. Bernhard, "Directional modulation technique for phased arrays," IEEE Transactions on Antennas and Propagation, vol. 57, no. 9, pp. 2633-2640, Sep. 2009.

[7] H. Aggrawal, R. Puhl, and A. Babakhani, "Ultra-wideband pulse-based directional modulation," in 2015 IEEE MTT-S International Microwave and RF Conference (IMaRC), Hyderabad, India, Dec. 2015, pp. 292295.

[8] J. Sarrazin, M. Odhiambo, S. Golstein, P. De Doncker, and F. Horlin, "Spatial data focusing: An alternative to beamforming for geocasting scenarios," in 2018 USNC-URSI Radio Science Meeting (Joint with APS Symposium), Boston, MA, USA, Jul. 2018, pp. 139-140.

[9] G. Molineaux, S. Golstein, M. Odhiambo, F. Horlin, P. De Doncker, and J. Sarrazin, "Spatial data focusing using time and iq resources for wireless geocasting," in 2019 IEEE Global Communications Conference (GLOBECOM), Waikoloa, HI, USA, Dec. 2019.

[10] W.-Q. Wang, H. Shao, and J. Cai, "Range-angle-dependent beamforming by frequency diverse array antenna," International journal of antennas and propagation, vol. 2012, Aug. 2012.

[11] Y. Liu, H. Ruan, L. Wang, and A. Nehorai, "The random frequency diverse array: A new antenna structure for uncoupled direction-range indication in active sensing," IEEE Journal of Selected Topics in Signal Processing, vol. 11, no. 2, pp. 295-308, Mar. 2017.

[12] J. Hu, S. Yan, F. Shu, J. Wang, J. Li, and Y. Zhang, "Artificial-noiseaided secure transmission with directional modulation based on random frequency diverse arrays," IEEE Access, vol. 5, pp. 1658-1667, Jan. 2017. 